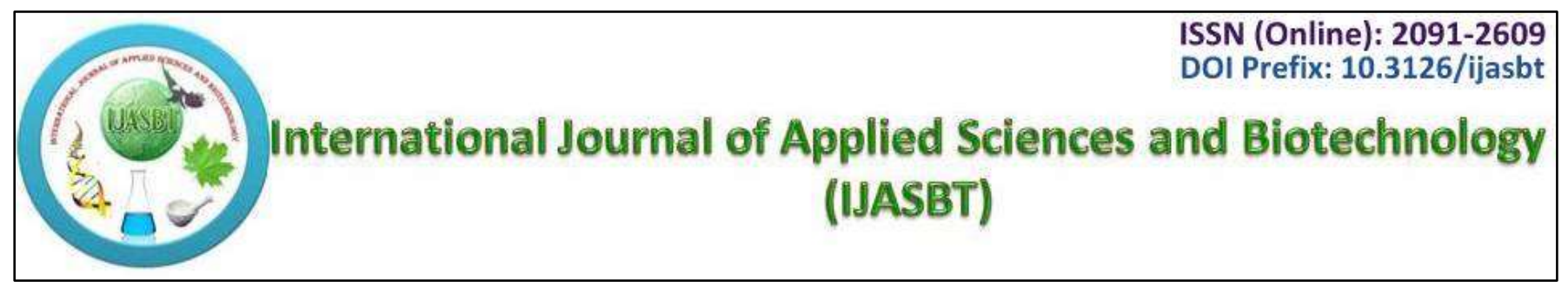

Research Article

\title{
Counteracting Congenital Cataract
}

\author{
Arthy Narayanan, B.V. Sai Sinduri and Pradeep $S^{*}$ \\ Department of Biotechnology, BMS College Of Engineering, Bangalore, India \\ *Corresponding Author’s email: padees@gmail.com
}

\begin{abstract}
Congenital or hereditary cataract is a condition where the lens of the eye is not completely transparent resulting in cloudy or blocked vision. Many genes have been found to influence the formation of a cataract. The gene that has been focused on in this paper is the CRYAA gene which codes for the crystallin A protein, a major constituent protein of the lens. Mutations and misfoldings of this protein have been seen to be a major cause of congenital cataract. So, we did an analysis of the gene and the protein using computational tools. As a solution to the condition, we computationally devised a vector that carries the correct CRYAA gene. This vector could be used during the pre-natal stage of pregnancy for children who are believed to inherit this condition.
\end{abstract}

Keywords: CRYAA; Alpha A Crystallin; Congenital cataract

\section{Introduction}

Congenital cataract is a hereditary condition where the lens of the eye becomes cloudy, making it difficult to see. This condition is due to the clumping of denatured proteins in the lens (Kinoshita, 1974). As of now, the diagnosis is by an eye examination and the current treatment for this is surgery where the lens is removed and replaced by an artificial one.

The lens of the eye is a clear tissue which focuses the light per its shape. The ciliary muscles and fibres contract or relax which results in the stretching or relaxing of the lens. This stretching and relaxing of the lens gives it different conformations, which will refract light differently. If the lens does not focus the light correctly onto the retina, then some of the visual information is lost. If the lens is unable to change its shape, it results in far-sightedness or closesightedness. If the lens becomes cloudy, it results in cataract (Cheng, 1989; Usha P. Andley, 2008).

The lens is made up of three layers. They are the outer capsule layer, the middle epithelial layer and the inner axial fibers. If the lens were to be cut longitudinally, the axial fibers would look like onion rings. The outer capsule is made up of collagen and provides plasticity to the lens. The inner axial fibers do not contain nuclei. They are mostly made up of proteins which contribute to the refractive index of the lens. The middle epithelial layer is the precursor to the axial fiber layers. This middle layer has cells with nuclei which make the protein. As this layer gets older, its cells start to lose their nuclei and they form a new layer of the axial fibers (Andley, 2008).

The layers of the axial fibers do not have protein turn-over. This means that they do not produce new protein. So, the protein found in the inner most axial fiber was formed during embryogenesis. Also, the proteins do not travel from one layer to another. They are restricted to their own layer (Andley, 2008).

During the natural aging process, the lens proteins begin to denature. When these proteins denature, they tend to aggregate and become insoluble. When the soluble proteins become insoluble, they reduce the transparency of the lens. To prevent this, there is a protein called alpha crystallin, which binds to the denatured proteins and prevents them from aggregating and becoming insoluble. Since there are no insoluble proteins, the transparency of the lens is unaffected (Andley, 2008).

There are many genes that contribute to the proper formation of alpha crystallin. In the case of congenital cataract, there is a mutation in one or more of these genes resulting in aggregation of denatured proteins, forming a cataract (Augusteya, 2004). We looked at three important genes which are CRYAA, GJA3 and EPHA2.

Through many studies conducted, it has been found that out of the crystallin A proteins, alpha A crystallin is more 
important than alpha B crystallin, when it comes to lens transparency (Horwitz, 2003).

Since the proteins formed once in the lens are permanent and do not move across layers, we thought of correcting the formation of the crystallin proteins from the first step, that is during the prenatal stages. The formation of lens in a foetus is seen to begin at week 5 and complete at week 7 of pregnancy. If the foetus DNA could be obtained from the amniotic fluid, that could be tested for congenital cataract. If the condition is present or likely, we thought of introducing a vector in the lens cells that could suppress the malfunctioning gene and produce the functional CRYAA protein.

\section{Tools and Databases}

1. NCBI: We performed a search on CRYAA protein and obtained the FASTA sequence from the NCBI Protein database. We also obtained the sequences of our genes by searching for it on the NCBI Nucleotide database.

2. SWISS-MODEL: The structure of alpha crystallin has still not been determined, and therefore we used a link to SWISS-MODEL provided in NCBI.

3. PROTEIN MODEL PORTAL: This tool provides the predicted 3D structure of the protein. The link to the 3D structure of alpha crystallin was given in NCBI.
4. RASMOL: We used this tool to visualize the A chain of alpha crystallin.

5. PROSITE: Here, we can see the domains and motifs of the proteins and understand the functions of the proteins. We got one hit for alpha crystallin in this database.

6. UNIPROT: We used UniProt to obtain gene sequences.

7. GENtle: We used this software to create our vector as a solution to congenital cataract.

\section{Results and Discussion}

FASTA Sequence of Alpha Crystallin, a protein, was obtained by NCBI protein database. The obtained sequence is show Fig. 1. Thereafter, protein model was obtained using SWISS-MODEL (3D) prediction of Alpha Crystallin A. by link provided in NCBI. The obtained model is illustrated in Fig. 2.

Fig. 3 shows the 3D structure of Alpha A Crystallin using RasMol viewer. In the command line, it was confirmed that the protein was the A chain of Alpha A crystallin and it was a chaperone.

Prosite tool is used generally to see the domains and motifs of the proteins and understand the functions of the proteins. We used this tool to find out the functions and domains of Alpha Crystallin A protein. One hit was obtained for Alpha A Crystallin (Fig. 4). Fig. 5 shows NCBI search result for CRYAA gene sequence.

Fig. 1: FASTA sequence of Alpha Crystallin, a protein

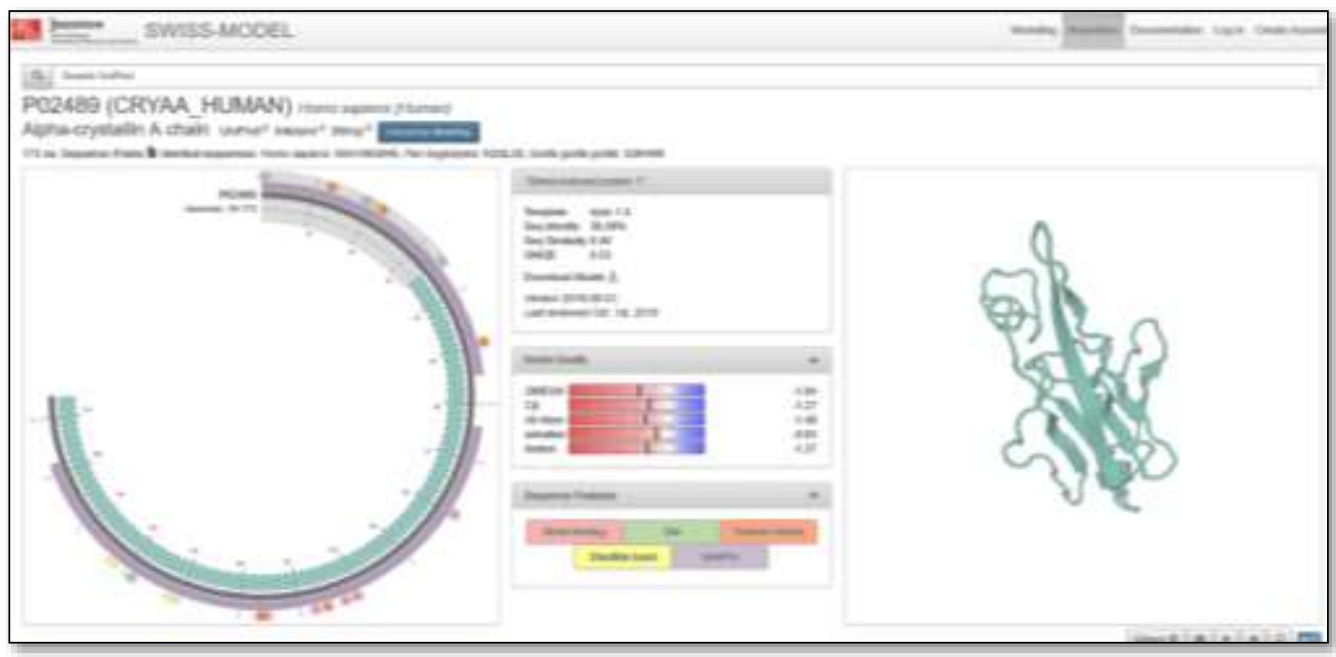

Fig. 2: SWISS-MODEL 3D prediction of Alpha Crystallin A. 


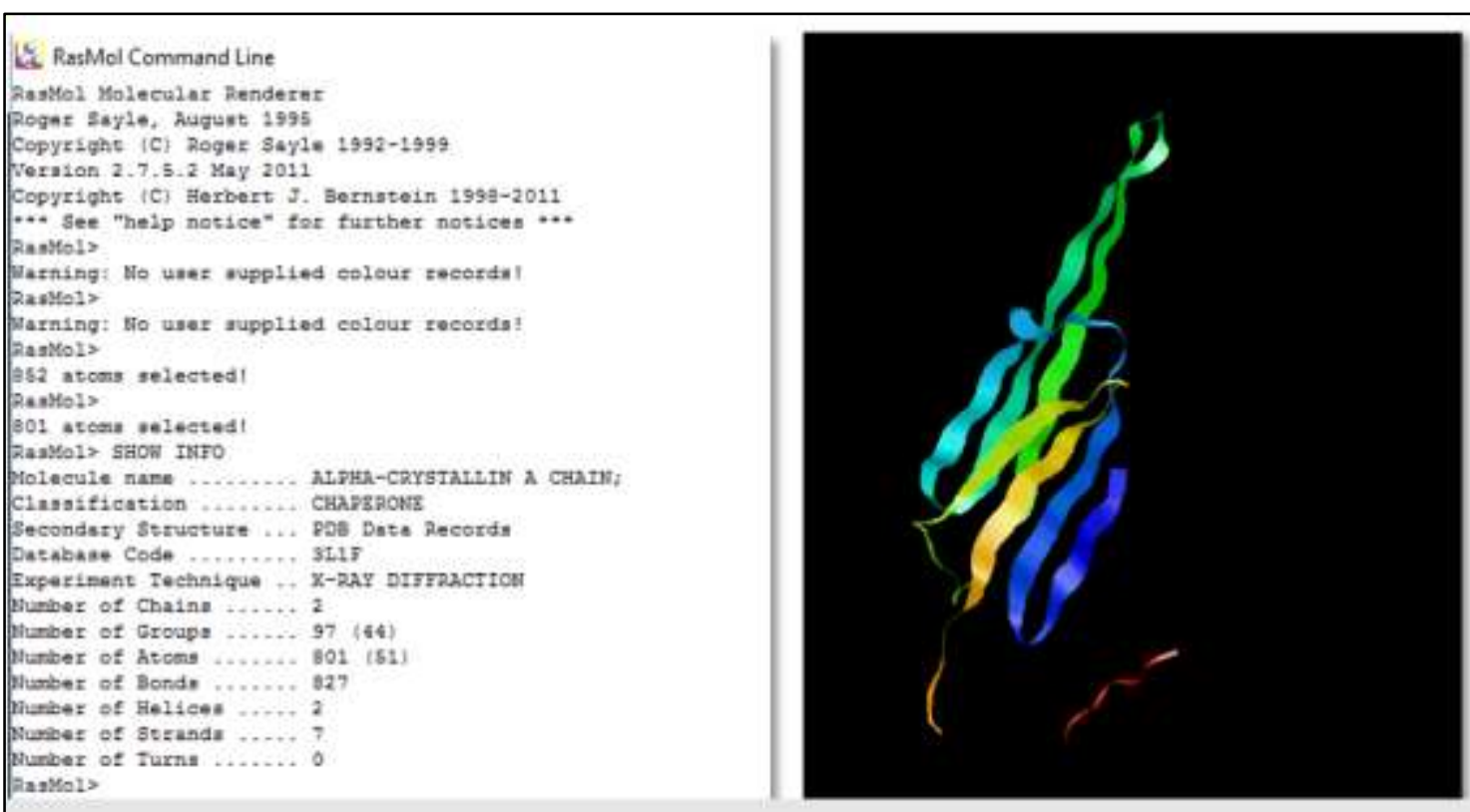

Fig. 3: RasMol viewer used to visualize the 3D structure of Alpha A crystallin.

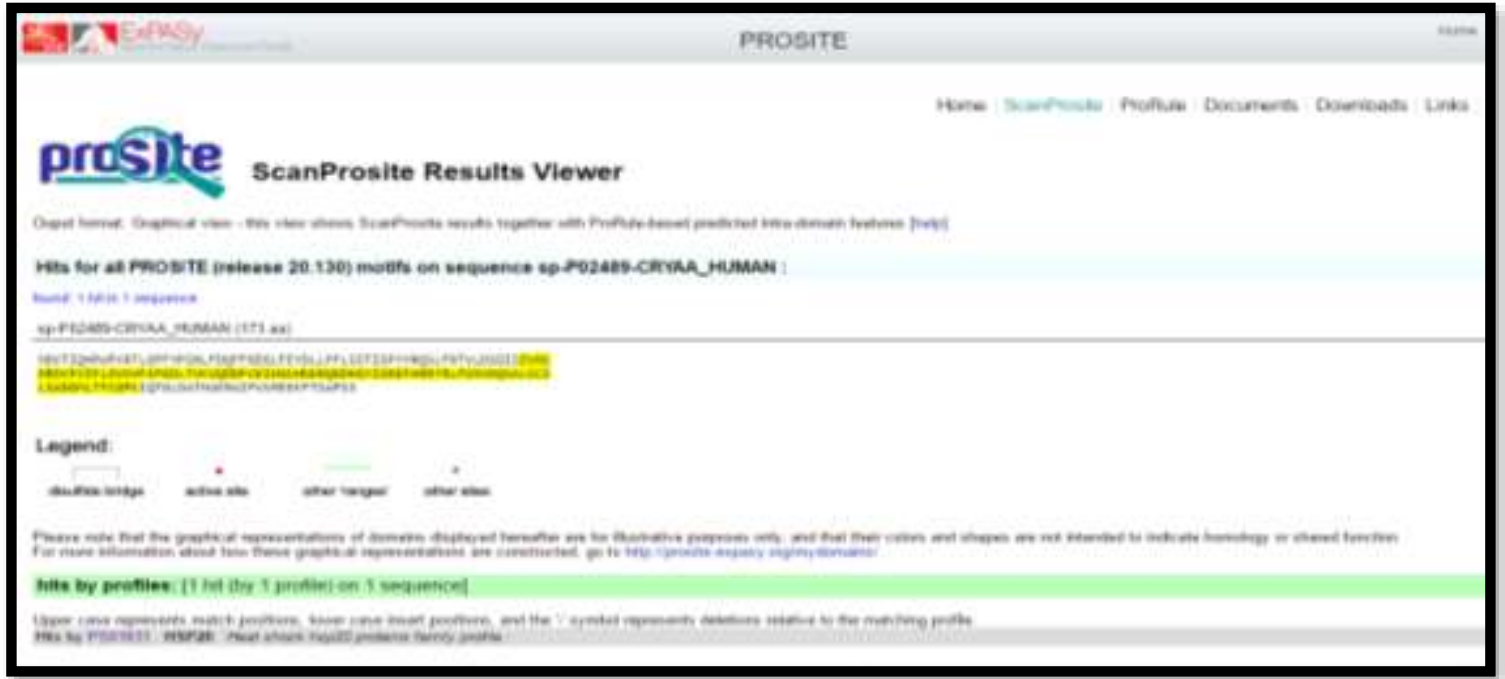

Fig.4: Prosite was used to get the function and domains of the protein.

\begin{tabular}{|c|c|c|}
\hline \multicolumn{3}{|c|}{ 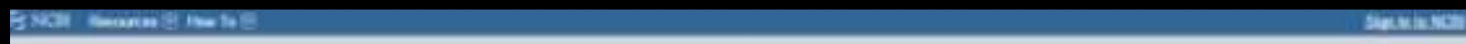 } \\
\hline \multirow[t]{2}{*}{ Pente } & \multirow{2}{*}{\multicolumn{2}{|c|}{5}} \\
\hline & & \\
\hline \multicolumn{3}{|c|}{ 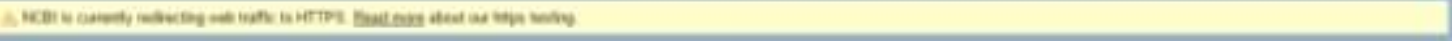 } \\
\hline \multicolumn{2}{|l|}{ vilient: } & cenesen \\
\hline \multirow{2}{*}{\multicolumn{2}{|c|}{ 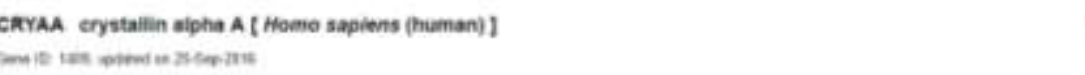 }} & beserinements \\
\hline & & Semer \\
\hline - Summir & & theove unsur \\
\hline \multirow{3}{*}{ 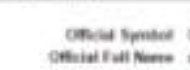 } & & 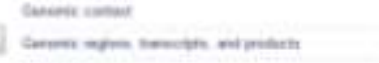 \\
\hline & omou nombers & manernt \\
\hline & 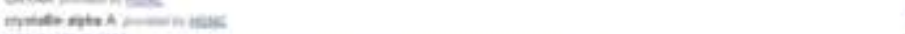 & nensum \\
\hline \multirow{2}{*}{ 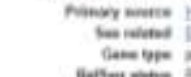 } & 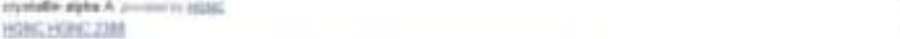 & Wowe \\
\hline & 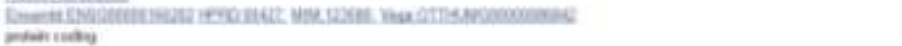 & 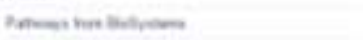 \\
\hline Gethey athe : & WNeines & heresties \\
\hline aganam & 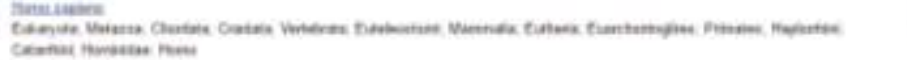 & 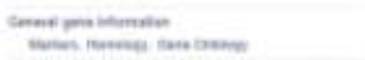 \\
\hline \multirow{5}{*}{ Mes inen is } & 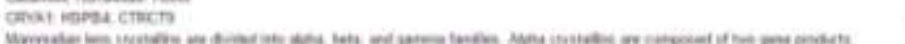 & 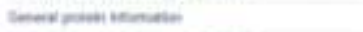 \\
\hline & \multirow{4}{*}{ 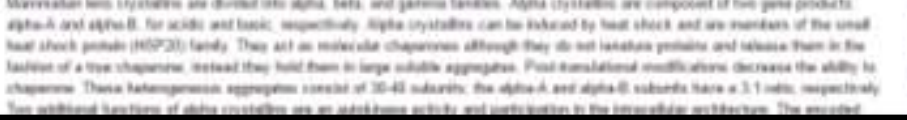 } & 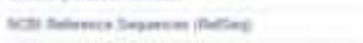 \\
\hline & & Moviot nepmese \\
\hline & & sasenest \\
\hline & & \\
\hline
\end{tabular}

Fig. 5: NCBI search result for CRYAA gene sequence 
The amino acid sequence of Alpha Crystallin A was pasted into a tool GENtle for plasmid mapping. GENtle is a free software for DNA and amino acid editing, database management, plasmid maps, restriction and ligation, alignments, sequencer data import, calculators, gel image display, PCR, and much more. The obtained result is shown in Fig. 6.
Fig. 7 shows the result of back-translation of the amino acid sequence to a DNA sequence using the option 'Backtranslate DNA' in GENtle.

The obtained final human DNA sequence after back-translation is shown in Fig. 8.

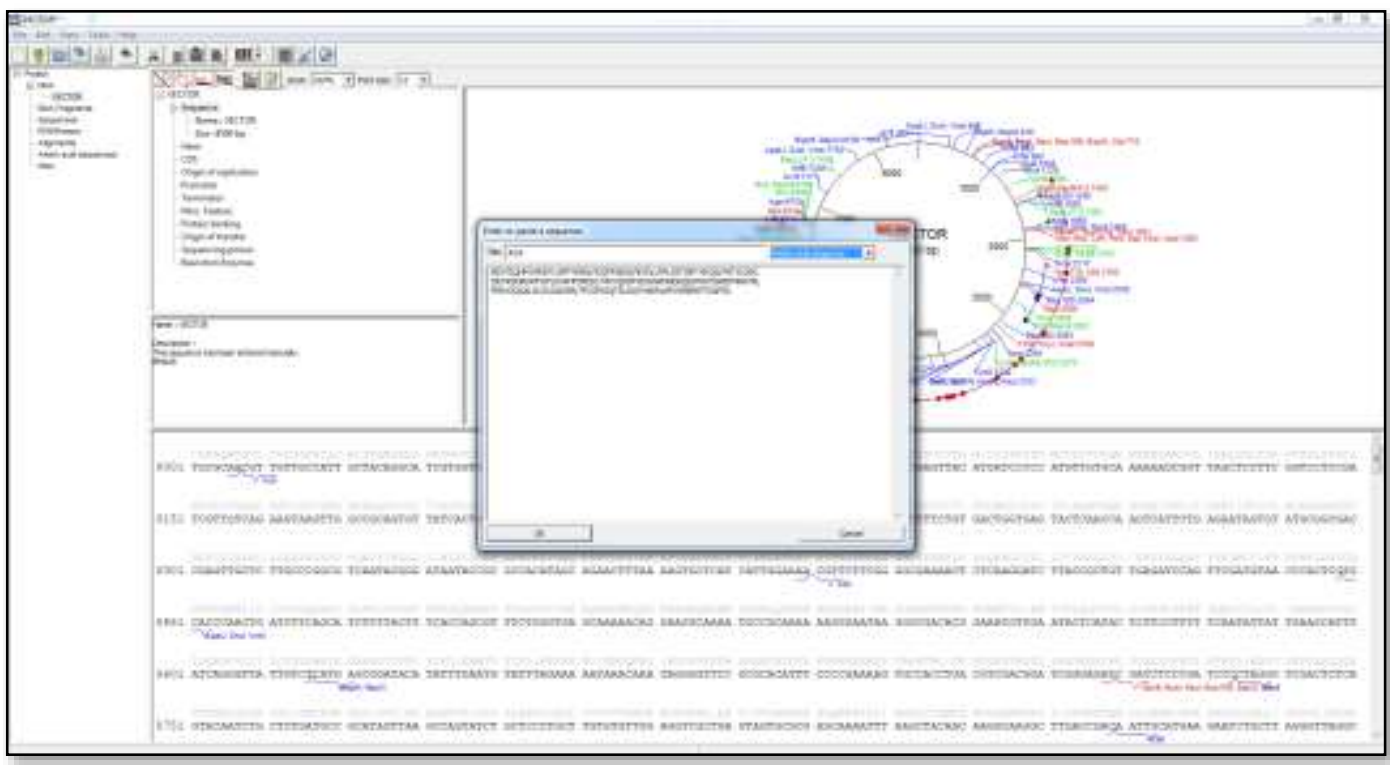

Fig. 6: We pasted the amino acid sequence of Alpha Crystallin A into GENtle

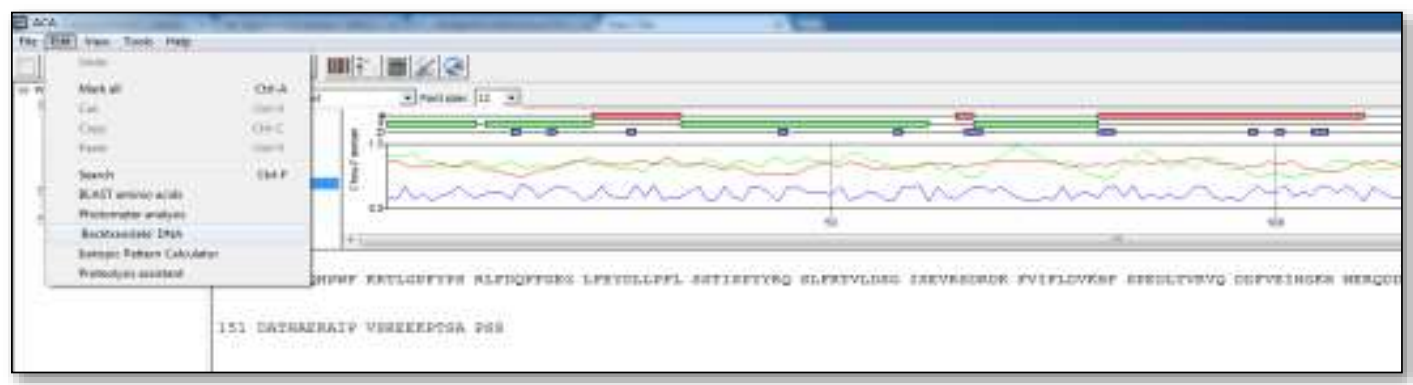

Fig. 7: In GENtle we back-translated the amino acid sequence to a DNA sequence.

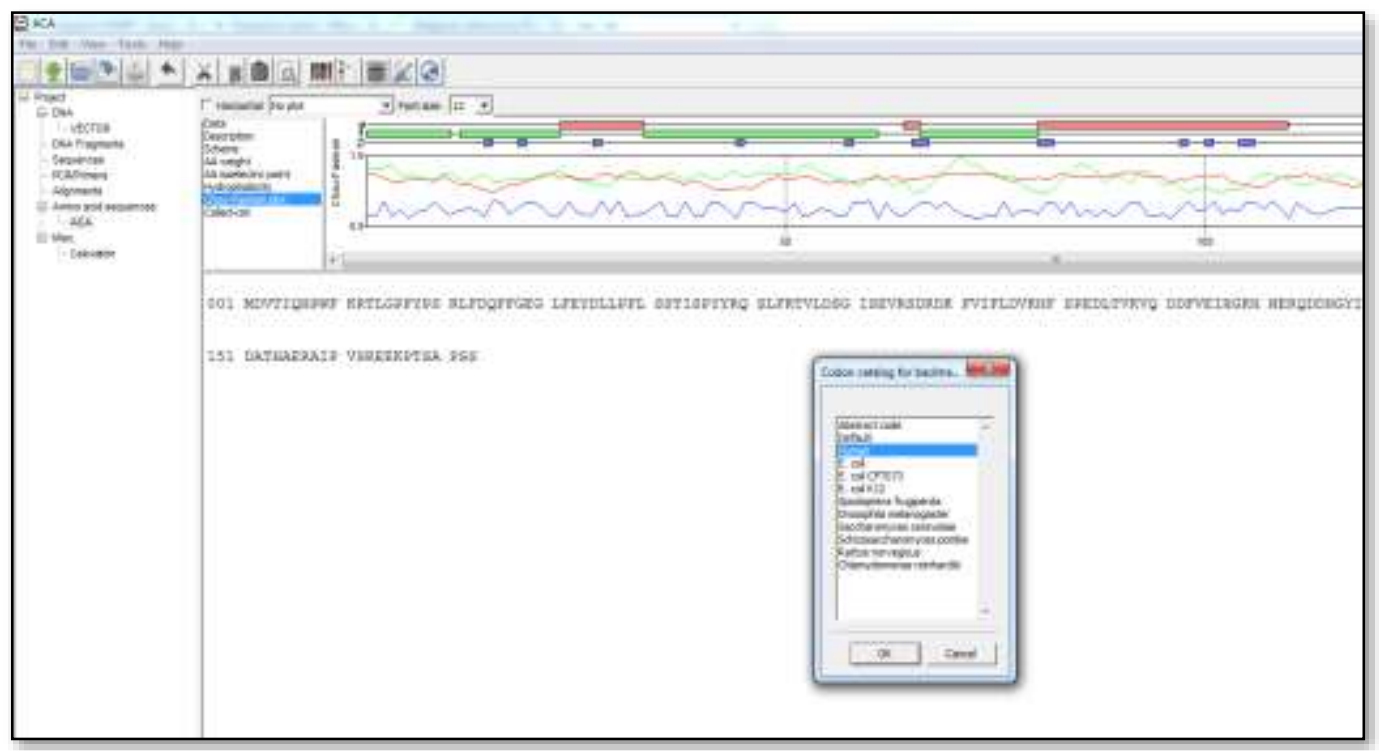

Fig. 8: The resulting human DNA sequence. 
Fig. 9 shows the result of adding an adaptor having an EcoRI site to the beginning and end of the sequence. An adaptor is added to ligate the ends of a DNA sequence with another DNA sequence or a vector. Addgene' is a nonprofit plasmid repository, we used this repository to obtain vector.

Fig. 10 shows a mammalian vector chosen from Addgene.

We restricted and ligated the vector and gene sequence Fig. 11 shows the restriction and ligation of the vector and the gene sequence using the respective options in GENtle.

We obtained our final vector displayed in GENtle (Fig. 12), that encodes the gene to code Alpha A crystallin. The gene sequence was obtained from NCBI and should code for a functional protein, which would be able to prevent the aggregation of insoluble proteins. All this work has been done computationally. This computational vector must be produced and tested in human or animal lens cells to check if the alpha A crystallin is being formed properly. There may be other factors which need to be added for the proper formation of functional alpha A crystallin. Once it has been confirmed that alpha A crystallin is being formed, it must be checked whether these proteins are sufficient to prevent cataract. This could be done initially using animal models.
An inducer may need to be added to the vector to suppress the formation of non-functional alpha A crystallin by the host genome.

The first step in generating an effective cure for any condition is to know its cause. Similarly, for congenital cataract there can be many potential causes such as mutations in CRYAA, GJA3 and EPHA2 genes, environmental effects, etc (Cheng, 1989). The cause portrays the nature of the cataract and the ways it can be prevented or controlled. In the case of genetic effects, knowing the cause is necessary for ideal vector construction. In our experiment, we have considered the improper formation of Alpha A Crystallin as the cause of the cataract. To overcome this, we have computed a vector which can code for a functional Alpha A Crystallin protein.

This vector is a solution to the immediate problem, which is the non-functional crystallin protein. There could be many factors, which lead to the formation of a non-functional crystallin protein. Control or prevention of these factors could be looked at as an alternative treatment or cure.

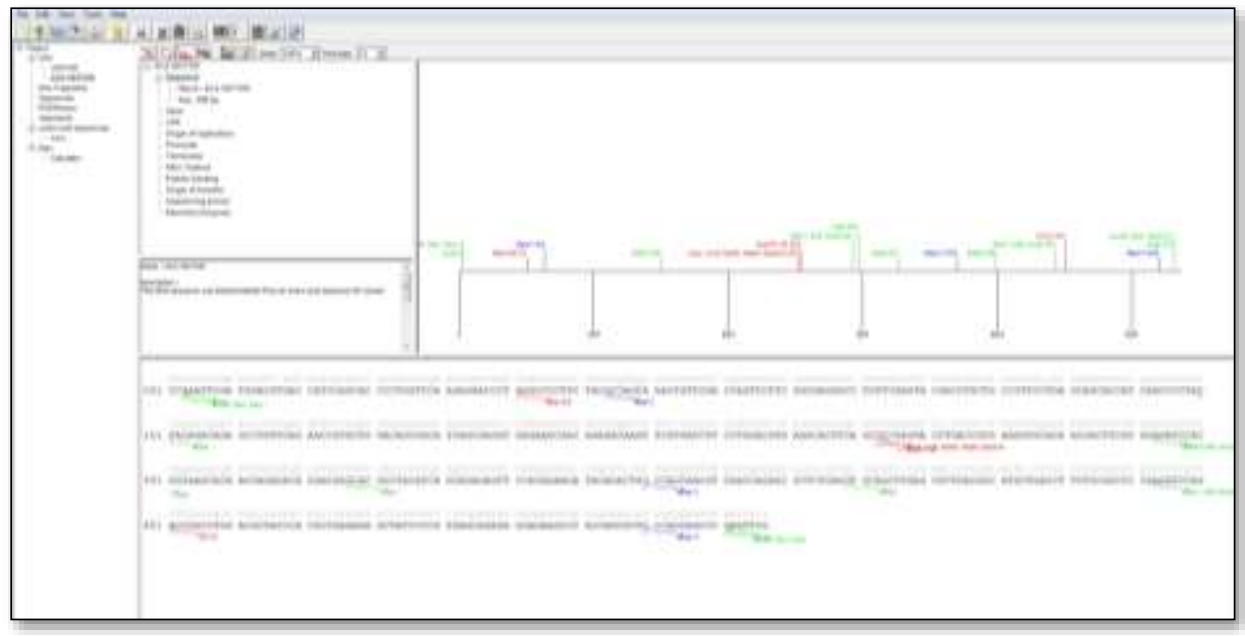

Fig. 9: The result of adding an adaptor having an EcoRI site to the beginning and end of the sequence.

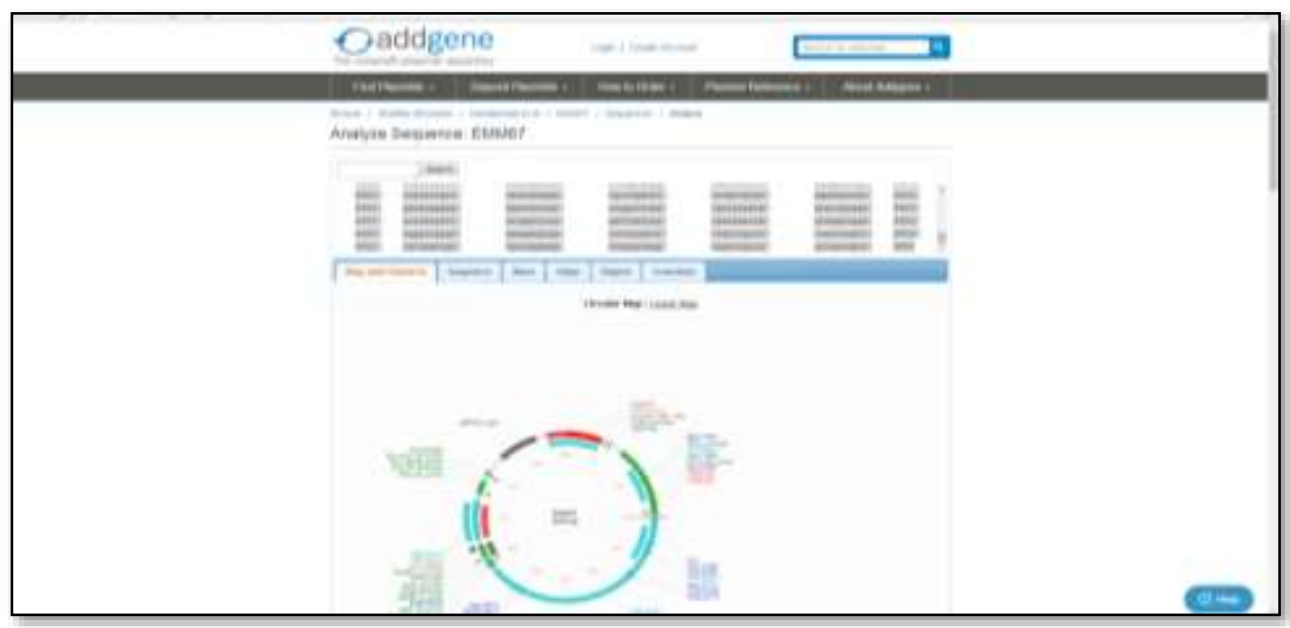

Fig. 10: We obtained a mammalian vector from addgene 


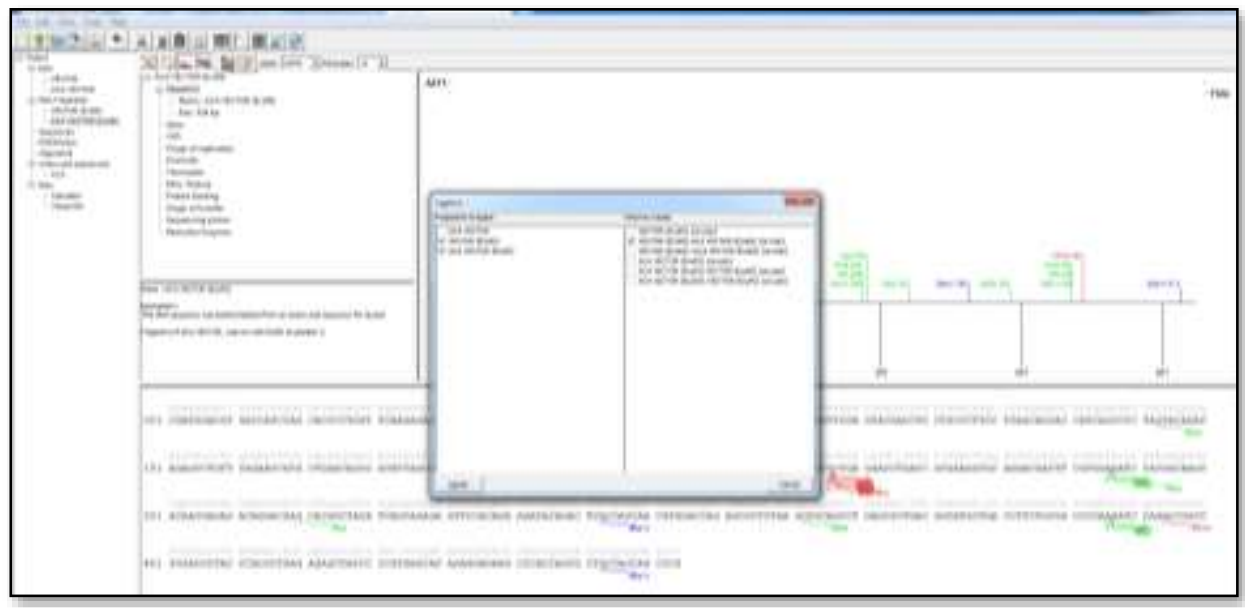

Fig. 11: Restricted and ligated vector and gene sequence by GENtle.

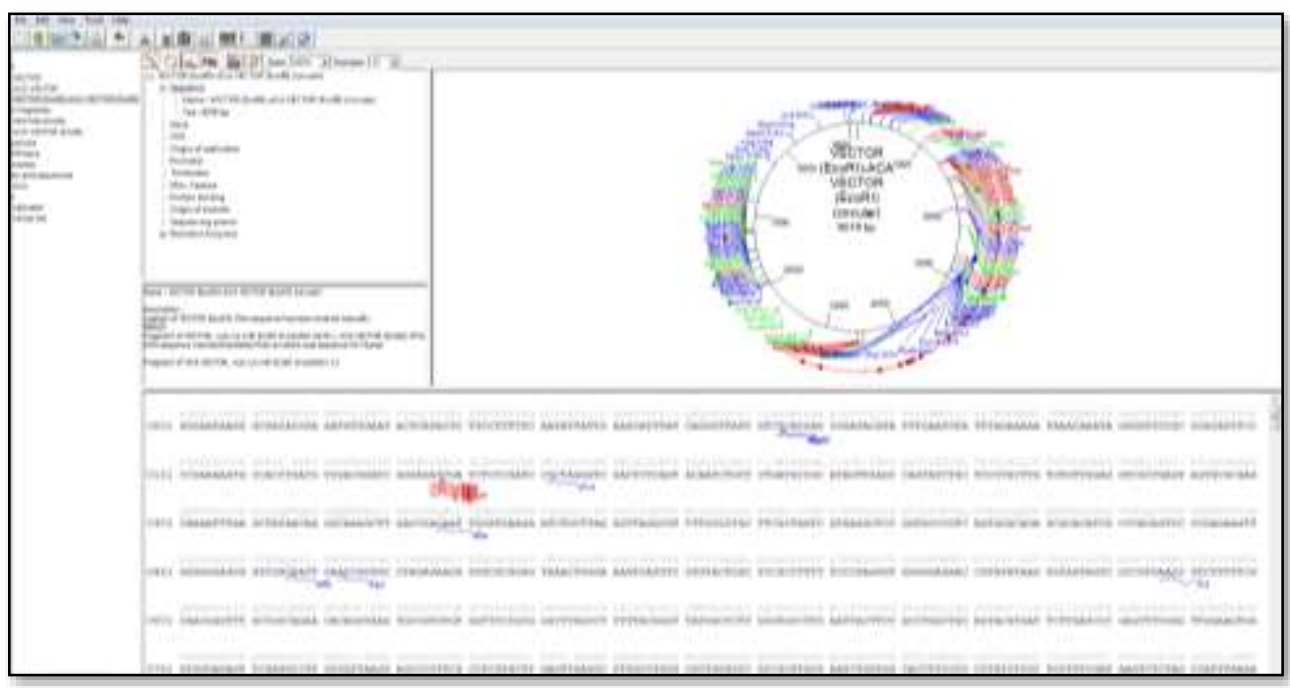

Fig. 12: Final vector obtained by GENtle.

\section{Conclusion}

From the work that we have done, we have obtained a vector that codes for a functional Alpha A crystallin. Since Alpha A crystallin formation begins after week 7 of pregnancy and goes on till death, making a vector seemed like the best solution for cataract. Another reason for using this vector is the fact that the proteins formed in each layer of the axial fibers of the lens are permanent and do not cross over to another layer. If this vector was used, it would be inserted into the lens cells at the end of week 7, right after testing the cff DNA (cell-free fetal DNA) for congenital cataract. This vector would produce functional Alpha A crystallin right from the start and each layer would have this functional protein. This should prevent cataract.

\section{Acknowledgement}

We would like to thank our HOD, Dr. Savithri Bhat, BMS College of Engineering, Department of Biotechnology and the lab assistants for providing us with the resources to conduct this project.

\section{References}

Andley UP (2008), The Lens Epithelium: Focus on the expression and function of the alpha-crystallin chaperones, Int $J$ Biochem Cell Biol 40(3): 317-323. DOI: $\underline{10.1016 / \text { j.biocel.2007.10.034 }}$

Augusteya RC (2004) $\alpha$ - crystallin: a review of its structure and function. Clin Exp Optom 87(6):356-366. DOI: 10.1111/j.1444-0938.2004.tb03095.x

Cheng H (1989) Causes of cataract. BMJ: British Medical Journal 298(6686): 1470. DOI: $10.1136 / \mathrm{bmj} .298 .6686 .1470$

Hejtmancik JF (2008) Congenital Cataracts and their Molecular Genetics. Semin Cell Dev Biol 19(2): 134-149. DOI: $\underline{10.1016 / j . s e m c d b .2007 .10 .003}$

Horwitz J (2003) Alpha-crystallin. Experimental Eye Research 76: 145-153. DOI: $10.1016 / \mathrm{S} 0014-4835(02) 00278-6$

Kinoshita JH (1974) Mechanisms initiating cataract formation proctor lecture. Investigative Ophthalmology \& Visual Science 13(10): 713-724. 